\title{
Calcul d'écoulements en domaines déformables : de l'ALE à la transpiration
}

\author{
Flow calculation for things subject to deformations : \\ from ALE to transpiration
}

par P. Le Tallec, M. Fernandez

Université Paris Dauphine et Ecole Polytechnique

Efficient formulations in fluid structure interaction are usually based on a purely lagrangian description of the structure coupled to an ALE formulation of the surrounding fluid. The present paper briefly reviews those formulations, describes their basic energy conservation properties, and the difficulties encountered in their numerical implementation. It then explains how an adequate asymptotic expansion can reduce such formulations to simplified models widely used in aeronautics, which combine the fundamental equations of fluid mechanics written in Eulerian coordinates with non standard transpiration interface boundary conditions.

\section{I —INTRODUCTION}

Le calcul d'écoulements autour de structures déformables (écoulements biologiques dans des tuyaux collabables, amortisseurs hydrauliques, écoulements autour de grands ouvrages et d'avions déformables) pose des problèmes difficiles de compatibilité cinématique, d'actualisation de géométrie, de conservation d'énergie cinétique, et d'efficacité numérique. Pour surmonter ces difficultés, la stratégie de référence consiste à traiter la structure et le fluide comme un milieu continu unique et à en rapporter le mouvement à la configuration de calcul délimitée par la structure au repos. Cette approche conduit ainsi à une modélisation purement lagrangienne de la structure, couplée à une formulation ALE du problème fluide. Cette formulation permet alors l'utilisation de schémas de discrétisation et d'algorithmes de couplage simples respectant la compatibilité cinématique à l'interface.

Cette formulation introduite en section II a de bonnes propriétés de conservation énergétique : une discrétisation raisonnable de cette formulation permet de bien prédire l'évolution globale de l'énergie cinétique du système et donc ainsi de donner un premier contrôle sur sa stabilité globale (sections III, IV).

Cependant, cette formulation nécessite d'introduire des maillages mobiles et de calculer des vitesses de grille, ce qui peut être délicat dans un environnement industriel, impli- quant des géométries complexes. Dans l'hypothèse où le mouvement de la structure reste modéré, les ingénieurs ont mis au point une technique qui permet de s'affranchir de ces problèmes de grilles mobiles et de se ramener par un changement approprié de conditions aux limites à un problème plus simple posé sur grille fixe. Nous verrons alors en section $\mathrm{V}$ comment obtenir mathématiquement cette nouvelle formulation par changement de variables et développement asymptotique.

\section{II — PROBLÈME MÉCANIQUE}

On considère dans cet article un système mécanique qui occupe un domaine variable $\Omega(t)$ dans sa configuration actuelle. Il est constitué d'une structure déformable (avion, ouvrage de génie civil) occupant le domaine $\Omega^{s}(t)$ et d'un fluide environnant occupant le complément $\Omega^{\prime}(t)$ dans $\Omega(t)$. Le problème consiste à prédire l'évolution en temps $x\left(\Omega_{0}, t\right)$ de cette configuration, de la vitesse du fluide $U(t)$, du tenseur des contraintes $\sigma(t)$ dans le fluide et dans la structure, et à estimer la stabilité globale du système étudié.

On supposera pour simplifier que le fluide est parfait. En introduisant les champs de vitesse $U^{s}=U_{\Omega_{\Omega^{s}}}$ et $U^{f}=U_{\Omega^{f}}$ dans la structure et dans le fluide, la condition de non pénétration à l'interface prend alors la forme cinématique :

$$
\operatorname{Tr}\left(U^{s}\right)_{\mid \Gamma} \cdot n(t)=\operatorname{Tr}\left(U^{t}\right)_{\mid \Gamma} \cdot n(t),
$$




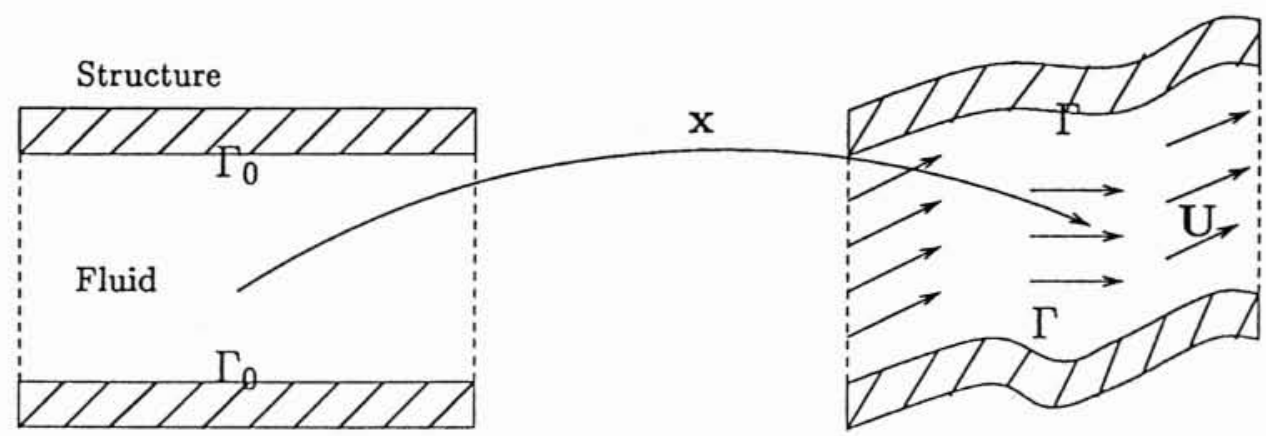

Reference config. $\Omega_{0}$

Present config. $\Omega(t)$

1. Configurations géométriques : la configuration de référence fixe $\Omega_{0}$, dans laquelle la structure est délimitée par la structure au repos, et la configuration physique $\Omega(t)$ à l'instant $t$.

où $n(t)$ dénote la normale à l'interface fluide structure en configuration actuelle dirigée vers l'intérieur de la structure, et $\operatorname{Tr}$ la restriction du champ de vitesses à cette même interface. Dans ce cadre, l'évolution de la densité, de la vitesse, et des contraintes est gouvernée par les lois de conservation usuelles écrites en configuration actuelle $\Omega(\mathrm{t})$. Mais comme cette configuration dépend du temps, il est plus pratique de se ramener à une configuration de référence fixe $\Omega_{0}$ délimitée par exemple par la position d'équilibre de la structure. Pour ce faire, il suffit d'introduire une transformation continue :

$$
\begin{array}{r}
x: \Omega_{0} \times \Re \rightarrow \Omega(t), \\
\left(x_{0}, t\right) \rightarrow x\left(x_{0}, t\right),
\end{array}
$$

qui envoie tout point $x_{0}$ de la configuration fixe $\Omega_{0}$ vers son image $x\left(x_{0}, t\right)$ en configuration actuelle $\Omega(t)$, et d'introduire les taux de variation $\frac{\partial f}{\partial t} x_{t}$ des quantités physiques $f$ à la position $x_{0}$ fixée en configuration fixe. Le choix de la configuration $\Omega_{0}$ et de la carte $x$ peut être arbitraire, ce qui justifie l'appellation "Arbitrary Lagrangian Eulerian " (ALE) donnée à la formulation qui en résulte. Pour simplifier les calculs de structure, il est cependant préférable d'utiliser une configuration matérielle pour la structure $[4,5]$. Autrement dit, sur la structure $\Omega^{s}$, le point $x\left(x_{0}, t\right)$ correspond à la position actuelle $x^{5}(t)$ du point matériel qui se trouvait en $x_{0}$ à l'instant $t_{0}$. Cela implique en particulier que la vitesse de

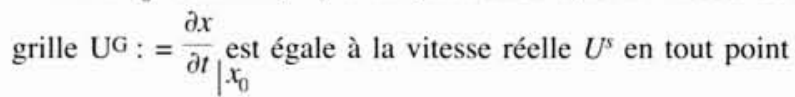
de la structure $\Omega^{s}$. Par contre, le choix de la carte fluide $x^{f}$ de $\Omega_{0}^{f}$ sur $\Omega^{f}(t)$ reste arbitraire en tout point intérieur de la grille de discrétisation du domaine fluide. Il peut s'agir de toute extension raisonnable $x^{f}=\operatorname{Ext}\left(x_{\Gamma_{\Gamma_{0}}}^{f}\right)$ qui se recolle au mouvement de la structure sur l'interface :

$$
x^{f}=\operatorname{Ext}\left(x_{\mid \Gamma_{0}}^{f}\right), \frac{\partial x^{f}}{\partial t} \mid \mathrm{r}_{0}=T_{r}\left(U^{s}\right)_{\mid \mathrm{r}} .
$$

La forme faible des lois de conservation peut alors se transporter sur la configuration de référence $\Omega_{0}$. En particulier, le travail virtuel des forces d'interface se transporte en :

$$
\int_{\Gamma_{(t)}} p n \cdot\left(\hat{U}^{f} \pm \hat{U}^{s}\right) d a=\int_{\Gamma_{0}} J_{p}\left(F^{ \pm T} \cdot n_{0}\right) \cdot\left(\hat{U}^{f} \pm \hat{U}^{s}\right) d a_{0}
$$

sous la notation:

$$
F=\frac{\partial x}{\partial x_{0}}, J=\operatorname{det} F, \rho_{0}=J \rho .
$$

Il faut ensuite prendre en compte les différentes lois de comportement. Sur la partie fluide, il est intéressant de revenir à la configuration actuelle pour calculer les termes de contraintes. Pour la structure, il convient de rester en configuration matérielle. En effet, pour le cas de structures élastiques en grandes déformations, le second tenseur des contraintes de Piola Kirchhoff $S=J F^{ \pm 1} \cdot \sigma \cdot F^{ \pm T} \quad$ s'y

écrit en fonction de la densité d'énergie élastique $\varphi\left(x_{0}, E\right)$ et les intégrales de masse et de raideur se réduisent alors à :

$$
\begin{aligned}
& m^{2}(\ddot{x} s, \hat{U}):=\int_{\Omega_{0}^{s}} \rho_{0} \ddot{x^{s}} \cdot \hat{U}, \\
& a^{s}\left(x^{s}, \hat{U}\right):=\int_{\Omega_{0}^{s}} F \cdot S: \frac{\partial \hat{U}}{\partial x_{0}}=\int_{\Omega_{0}^{s}} \frac{\partial}{\partial \underline{E}} \varphi\left(x_{0}, \underline{E}\right): \underline{\underline{E}}(\hat{U}),
\end{aligned}
$$

l'accélération $\ddot{x}^{s}$ et le tenseur $\underline{E}(\hat{U})$ des taux lagrangiens de déformation virtuelle de la structure étant respectivement définis par:

$$
\ddot{x}^{s}=\dot{U}^{s}=\frac{\partial^{2} x^{s}}{\partial t^{2}} x_{0}, \underline{\hat{E}}(\hat{U})=\frac{1}{2}\left(F^{T} \cdot \frac{\partial \hat{U}}{\partial x_{0}}+\frac{\partial \hat{U}^{T}}{\partial x_{0}} \cdot F\right) .
$$

Les formulations restent similaires si on utilise des modèles de poutres ou de coques en grands déplacements [9].

Tous ces calculs conduisent finalement à une formulation globale des lois de conservation de masse et de quantité de mouvement sur l'ensemble fluide structure, de la forme :

$\int_{\Omega_{0}} \frac{\partial J \rho}{\partial t_{\mid x 0}} \cdot \hat{q}+\int_{x\left(\Omega_{0}, t\right)} d i v_{x}\left(\rho\left(U \pm U^{G}\right)\right) \hat{q}=0, \forall \hat{q}: \Omega_{0} \rightarrow \Re$, (Masse $)$,

$$
\begin{aligned}
& m^{s}\left(\ddot{x^{s}}, \hat{U}\right)+\int_{\Omega} \frac{\partial J \rho U^{f}}{\partial t} \cdot \hat{U}+\int_{x f\left(\Omega_{0}^{f}, t\right)} \operatorname{div}_{x}\left(\rho U^{f} \hat{U}\left(U^{f} \pm U^{G}\right)\right) \cdot \\
& +a^{s}\left(x^{s}, \hat{U}\right) \pm \int_{x^{f}(\Omega f, t)} p \operatorname{div}\left(\hat{U}^{f}\right)+\int_{x} f\left(T_{0}, t\right) \\
& =\int_{\Omega(t)} f \cdot \hat{U}+\int_{\partial \Omega(t)} g \cdot\left(\hat{U}^{f} \pm \hat{U}^{s}\right) d a
\end{aligned}
$$

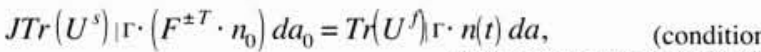

$x^{f}=\operatorname{Ext}\left(x_{\mid \Gamma_{0}}^{f}\right), \frac{\partial x^{f}}{\partial t} \mid \Gamma_{0}=\operatorname{Tr}\left(U^{s}\right)_{\mid \Gamma_{0}}, \quad \begin{array}{r}\text { cinématique d'interface),(5) } \\ \text { (mouvement de la grille fluide) }\end{array}$ 
Ces équations permettent de complètement prédire l'évolution du système étudié, quand on les complète par une équation d'état $p=g(\rho)$ reliant pression hydrodynamique $p$ et densité $\rho$ à l'intérieur du fluide, et par des conditions aux limites et initiales adéquates. En fait, pour des fluides compressibles où la loi d'état fait intervenir la température du fluide, il faut rajouter une équation de conservation d'énergie qui gouverne l'énergie totale $E=\rho e+\frac{1}{2} \rho U^{2}$ du fluide par :

$$
\begin{aligned}
& \int_{\Omega f} \frac{\partial J E}{\partial t \mid x_{0}} \hat{q} \pm \int_{x f\left(\Omega f_{0}, t\right)}\left(E\left(U \pm U^{G}\right)+p U^{f}\right) \cdot \frac{\partial \hat{q}}{\partial x} \\
& +\int_{\partial \Omega f(\theta)}^{?}\left(E\left(U \pm U^{G}\right)+p U^{f}\right) \cdot n \hat{q}=0, \forall \hat{q}: \Omega_{0}^{f} \rightarrow \Re,(\text { Energie })
\end{aligned}
$$

\section{III —DISCRÉTISATION}

La résolution numérique des équations passe d'abord par une discrétisation en temps des lois de conservation, devant respecter la compatibilité cinématique à l'interface et les grands principes de conservation d'énergie. Un choix simple mais grossier consiste à intégrer fluide et structure de manière décalée afin de pouvoir découpler la résolution numérique de ces deux problèmes : à chaque pas de temps, on résout d'abord le problème fluide à géométrie $x^{f}=x_{n}^{s}$ et vitesse de structure :

$$
\left(\operatorname{Tr} U_{\mid \Gamma}^{f}\right)_{n+1} \cdot n=\left(\operatorname{Tr} U_{n_{\Gamma}}^{s}\right) n
$$

imposées, puis on déduit une force d'interface $p_{n+1} n d a$ qui permet alors de résoudre le problème de structure à force d'interface imposée, afin de prédire la position et la vitesse finale d'interface $\left(x_{n+1}^{s}\right)_{\mid \mathrm{r}}$ et $\left(U_{n+1}^{s}\right)_{\mathrm{r}}$ pour le prochain pas de temps. Dans ce choix, la force d'interface $p n$ travaille sur le champ de vitesses $U_{n+1}^{f}=U_{n}^{s}$ côté fluide et sur le champ $U_{n+1}^{s} \neq U_{n}^{s}$ côté structure. Les puissances développées ne s'annulent pas, et il y a création artificielle d'énergie à l'interface.

Cette erreur peut être fortement réduite en améliorant la qualité de la prédiction de la vitesse d'interface $U_{n+1}^{f}$ dans

le calcul fluide [3]. Une autre possibilité est d'utiliser des schémas qui vérifient par construction un principe de conservation d'énergie cinétique [5]. Nous nous concentrons ici sur des schémas couplés à un point qui résolvent le problème couplé complet (3)-(6)(y compris les conditions de compatibilité cinématique) à l'interface aux instants $t, n=, \ldots$, en utilisant des approximations indépendantes des diverses dérivées en temps. Côté structure, le choix de référence qui combine précision, stabilité et absence de dissipation est un schéma de point milieu d'inconnue $x_{n+1 / 2}^{s}$ défini par [1]:

$$
\begin{gathered}
x_{n}^{s}=\frac{x_{n+1 / 2}^{s}+x_{n \pm 1 / 2}^{s},}{2}, \\
F_{n}=\frac{\partial x_{n}^{s}}{\partial x_{0}}, \hat{E}_{n}(\hat{U})=\frac{1}{2}\left(F_{n}^{T} \cdot \frac{\partial \hat{U}}{\partial x_{0}}+\frac{\partial \hat{U}^{T}}{\partial x_{0}} F_{n}\right) \\
S_{n}^{s}=\frac{1}{2}\left(S^{s}\left(x_{n+1 / 2}^{s}\right)+S^{s}\left(x_{n \pm 1 / 2}^{s}\right)\right) \\
U_{n}^{s}=\frac{x_{n+1 / 2}^{s} \pm x_{n \pm 1 / 2}^{s}}{\Delta t_{n}}=\frac{1}{2}\left(U_{n+1 / 2}^{s}+U_{n+1 / 2}^{s}\right), \\
\left(\ddot{x}^{s}\right)_{n}=\frac{U_{n+1 / 2}^{s} \pm U_{n \pm 1 / 2}^{s}}{\Delta t_{n}} .
\end{gathered}
$$

L'accélération du fluide peut être approchée par un simple schéma d’Euler implicite :

$$
\left(\frac{\partial \rho J U^{f}}{\partial t}\right)_{n}=\frac{\left(\rho J U^{f}\right)_{n} \pm\left(\rho J U^{f}\right)_{n \pm 1}}{\Delta t_{n}},
$$

par des schémas de type Runge Kutta, ou par un schéma de différences finies rétrograde de Gear :

$$
\left(\frac{\partial \rho J U^{f}}{\partial t}\right)_{n}=\frac{3}{2 \Delta t}\left(\rho J U^{f}\right)_{n \pm \frac{2}{\Delta t}}\left(\rho J U^{f}\right)_{n \pm 1}+\frac{1}{2 \Delta t}\left(\rho J U^{f}\right)_{n \pm 2} .
$$

\section{IV $\square$ CONSERVATION D'ÉNERGIE}

Le théorème de l'énergie cinétique est un outil fondamental dans l'analyse des problèmes d'interaction fluide structure, en particulier pour les problèmes à faible nombre de Mach. Pour ces problèmes, l'intégration en temps du principe de conservation de la quantité de mouvement multiplié par le champ de vitesse réelle indique que la variation de l'énergie totale du système (énergie cinétique totale plus énergie élastique de la structure) est égale à l'énergie introduite par les conditions aux limites externes diminuée de l'énergie dissipée par effets visqueux. Le respect de ce principe au niveau numérique garantit la stabilité et la précision à long terme de la procédure numérique, et permet de démontrer sous certaines conditions que la solution numérique converge vers la solution physique [2], [9].

La difficulté est que la plupart des schémas numériques violent ce principe quand on travaille sur des configurations mobiles $[5,11]$. On l'a déjà expliqué pour les schémas découplés. De manière plus surprenante, des schémas numériques totalement couplés qui sont précis et inconditionnellement stables quand on travaille sur des domaines fixes peuvent aussi perdre leurs propriétés de stabilité inconditionnelle en temps long quand le domaine de l'écoulement varie au cours du temps. Pour le voir, il suffit d'utiliser la forme faible de l'équation de conservation de quantité de mouvement (écrite de manière approchée au temps $t_{n}$ ) en prenant la vitesse réelle comme fonction test ( $U_{n}^{f}$ sur le fluide, $U_{n}^{s}$ sur la structure). Dans un schéma couplé, ce choix ne fait pas travailler les forces d'interface $p n$ sur $\Gamma$ car ces vitesses vérifient la condition de compatibilité cinématique (5). Sur la structure, l'action de $U_{n}^{s}$ donne bien la variation d'énergie cinétique quand il agit sur les termes d'inertie :

$$
\begin{aligned}
\left(\frac{\partial U}{\partial t}\right)_{n} \cdot U_{n}^{s}= & \frac{U_{n+1 / 2}^{s} \pm U_{n \pm 1 / 2}^{s}}{\Delta t_{n}} \cdot \frac{U_{n+1 / 2}^{s}+U_{n \pm 1 / 2}^{s}}{2} \\
& =\frac{\left|U_{n+1 / 2}^{s}\right|^{2} \pm\left|U_{n \neq 1 / 2}^{s}\right|^{2}}{2 \Delta t_{n}},
\end{aligned}
$$

et la variation d'énergie élastique (à un terme d'ordre élevé près si l'énergie de déformation n'est pas quadratique) quand il agit sur les termes de raideur:

$$
\begin{gathered}
\frac{S^{s}\left(x_{n+1 / 2}^{s}\right)+S^{s}\left(x_{n \pm 1 / 2}^{s}\right)}{2} \cdot \hat{E}_{-n}\left(\frac{\left.U_{n+1 / 2+}^{s} U_{n \pm 1 / 2}^{s}\right)}{2}\right) \\
=\frac{S^{s}\left(x_{n+1 / 2}^{s}\right)+S^{s}\left(x_{n \pm 1 / 2}^{s}\right)}{2} \cdot \frac{E_{n+1 / 2} \pm E_{n \pm 1 / 2}}{\Delta t_{n}} \\
=\frac{1}{\Delta t_{n}}\left|\psi\left(E_{n+1 / 2}\right) \pm \psi\left(\underline{E}_{n \pm 1 / 2}\right)+\frac{\partial^{3} \psi}{\partial E^{3}}(\underline{E}) \cdot\left(E_{n+1 / 2} \pm E_{n \pm 1 / 2}\right)^{3}\right|
\end{gathered}
$$

Sur le fluide, après quelques manipulations algébriques et l'utilisation du principe de conservation de masse [10], l'action du champ de vitesse $U_{n}^{f}$ sur les termes d'inertie redonne la variation d'énergie cinétique, à deux erreurs près : 


$$
\begin{aligned}
I_{n}^{f}= & \int_{\Omega_{0}} \frac{1}{2}\left(\frac{\partial J_{\rho}\left|U^{f}\right|^{2}}{\partial t}\right)_{n} \\
& +\int_{\Omega_{0}^{f}}\left(\frac{\partial J \rho U^{f}}{\partial t}\right)_{n} \cdot U_{n}^{f} \pm \frac{1}{2}\left|U_{n}^{f}\right|^{2}\left(\frac{\partial J \rho}{\partial t}\right)_{n} \pm \frac{1}{2}\left(\frac{\partial J \rho\left|U^{f}\right|^{2}}{\partial t}\right)_{n} \\
& \int_{x^{f}\left(\Omega_{0} J_{n}\right)}\left(\frac{1}{2}\left|U_{n}^{f}\right|^{2} \pm \hat{q}\right)\left(\frac{1}{J_{n}}\left(\frac{\partial J p}{\partial t}\right)_{n}+\operatorname{div}_{x}\left[\rho\left(U^{f} \pm U^{G}\right)\right]_{n}\right) .
\end{aligned}
$$

La première erreur, concentrée sur la dernière ligne, correspond à une erreur de troncature en espace :

$$
e_{h}=\int_{\Omega f_{\left(t_{n}\right)}} \frac{\Delta t}{2} \underset{q h \in Q h f}{i n f}\left(\frac{1}{2}\left|U_{n}^{f}\right|^{2} \pm q_{h h}\right)\left(\frac{1}{J_{n}}\left(\frac{\partial J \rho}{\partial t}\right)_{n}+\operatorname{div}_{x}\left[\rho\left(U^{f} \pm U_{G}\right)\right]_{n}\right)
$$

qui est très petite si on choisit bien les espaces de pression, ou si on utilise des schémas vérifiant une condition de conservation locale de la masse (discrete Geometric Conservation Law) après discrétisation [8]. La seconde erreur, concentrée sur la seconde ligne, est proportionnelle à l'erreur de troncature en temps du schéma de discrétisation utilisé, le coefficient de proportionnalité étant fonction de la régularité en temps du jacobien $J$ de la déformation de grille. Autrement dit, toute variation brutale de $J$ conduit localement à de grosses erreurs. Ces deux erreurs peuvent être petites si on utilise des schémas précis sur des solutions et grilles régulières, mais sauf exception, on ne peut pas contrôler leur signe, et donc leur accumulation au cours du temps peut conduire à des instabilités artificielles.

\section{V —TRANSPIRATION}

La formulation ALE étudiée jusqu'ici a deux inconvénients pratiques. D'une part, elle exige le calcul préalable à chaque pas de temps d'une grille fluide $x^{f}$ et d'une vitesse de grille associée $U^{G}$, qui doivent être régulières en temps et en espace et qui doivent suivre les déformations de la structure. D'autre part, les vecteurs flux $F(W)$ (les termes à l'intérieur des opérateurs divergence) ne sont pas identiques à ceux utilisés pour des grilles fixes, ce qui oblige à transformer en profondeur les codes de calcul usuels [7].

Pour éviter ces inconvénients et résoudre à faible coût des problèmes d'interaction de fluides avec des structures modérément déformables, les ingénieurs aéronauticiens ont mis au point une technique de transpiration qui ne fait pas intervenir de nouvelle grille ou de nouveau flux, et que nous allons maintenant justifier mathématiquement. Pour simplifier, nous nous restreindrons au cas des problèmes stationnaires en temps. Le principe mathématique sous-jacent consiste à écrire le problème fluide sous forme variationnelle, en configuration actuelle $x^{f}\left(x_{0}\right)=x_{0}+\delta x\left(x_{0}\right)$, par rapport à l'inconnue :

$$
\delta W\left(x_{0}\right)=W\left(x^{f}\left(x_{0}\right)\right) \pm W_{0}\left(x_{0}\right) \pm \nabla W_{0}\left(x_{0}\right) \cdot \delta x\left(x_{0}\right) .
$$

Ici, $W$ dénote le vecteur caractérisant l'état du fluide ( $W^{t}=(\rho, \rho U, E)$ pour un fluide parfait), et $W_{0}$ est l'état du fluide quand la structure est dans sa configuration de référence. L'inconnue $\delta W\left(x_{0}\right)$ mesure donc l'écart entre la solution actuelle et la solution initiale au point physique $x^{f}\left(x_{0}\right)$. Elle vérifie les équations de conservation:

$$
\pm \int_{\Omega}^{f} F(W) \cdot \frac{\partial \hat{q}}{\partial x} d x+\int_{\partial \Omega}(F(W) \cdot n) \cdot \hat{q} d a=0, \forall \hat{q},
$$

qui, une fois transportées sur $\Omega_{0}$ s'écrivent :

$$
\begin{aligned}
& \pm \int_{\Omega_{0}^{f}} F\left(W_{0}+\nabla W_{0} \cdot \delta x+\delta W\right) \cdot \frac{\partial \hat{q} \partial x^{f}}{\partial x_{0}} \cdot\left(\frac{\partial x^{f}}{\partial x_{0}}\right)^{ \pm 1}\left|\frac{d x^{f}}{d x_{0}}\right| d x_{0} \\
& +\int_{\partial \Omega_{0}^{f}}\left(F\left(W_{0}+\nabla W_{0} \cdot \delta x+\delta W\right) \cdot n d a\right) \cdot \hat{q}\left(x_{0}+\delta x\right)=0, \forall \hat{q} .
\end{aligned}
$$

Pour exploiter cette équation, on lui retranche les équations de conservation satisfaites par la solution initiale au même point physique $x^{f}=x_{0}+\delta x$ (et non pas au point initial $x_{0}$ ):

$$
\pm \int_{\Omega_{0}} F\left(W_{0}\right) \cdot \frac{\partial \hat{q}}{\partial x} d x+\int_{\partial \alpha_{0}^{d}}\left(F\left(W_{0}\right) \cdot n_{0}\right) \cdot \hat{q} d a_{0}=0, \forall \hat{q},
$$

qui, transportées de $x^{f}$ à $x_{0}$, donnent :

$$
\begin{aligned}
& \pm \int_{\left(x^{\prime}\right)^{ \pm 1}\left(\Omega_{0}^{f}\right)} F\left(W_{0}\left(x_{0}+\delta x\right)\right) \cdot \frac{\partial \hat{q} o x^{f}}{\partial x_{0}} \cdot\left(\frac{\partial x^{f}}{\partial x_{0}}\right)^{ \pm 1}\left|\frac{d x^{f}}{d x_{0}}\right| d x_{0} \\
& +\int_{\partial \Omega_{0}^{f}}\left(F\left(W_{0}\right) \cdot n_{0}\right) \cdot \hat{q}\left(x_{0}\right) d a_{0}=0, \forall \hat{q} .
\end{aligned}
$$

Après linéarisation en $\delta x$, ce qui transforme la dernière équation en :

$$
\begin{gathered}
\pm \int_{\Omega_{0}^{f}} F\left(W_{0}\left(x_{0}+\delta x\right)\right) \cdot \frac{\partial \hat{q} o x^{f}}{\partial x_{0}} \cdot\left(\frac{\partial x^{f}}{\partial x_{0}}\right)^{ \pm 1}\left|\frac{d x^{f}}{d x_{0}}\right| d x_{0} \\
+\int_{\partial \Omega_{0}^{f}} F\left(W_{0}\right) \cdot \frac{\partial \hat{q}}{\partial x_{0}} \delta x \cdot n_{0} d a_{0}+\int_{\partial \Omega_{0}^{f}}\left(F\left(W_{0}\right) \cdot n_{0}\right) \cdot \hat{q}\left(x_{0}\right) d a_{0}=0, \forall \hat{q},
\end{gathered}
$$

et soustraction, on retrouve au premier ordre les équations d'Euler linéarisées :

$$
\begin{aligned}
& \pm \int_{\Omega_{0}^{f}}\left(\frac{\partial F}{\partial W}\left(W_{0}\right) \cdot \delta W\right) \cdot \frac{\partial \hat{q}}{\partial x_{0}} d x_{0} \\
& +\int_{\partial \Omega_{0}^{f}} \hat{q}\left(x_{0}\right)\left(\frac{\partial F}{\partial W}\left(W_{0}\right) \cdot\left(\nabla W_{0} \cdot \delta x+\delta W\right) \cdot n_{0} d a_{0}\right. \\
& +\int_{\partial \Omega_{0}^{f}} \hat{q}\left(x_{0}\right) F\left(W_{0}\right) \cdot\left(n d a \pm n_{0} d a_{0}\right)=0, \forall \hat{q} .
\end{aligned}
$$

Au premier ordre, en rajoutant les équations de conservation :

$$
\pm \int_{\Omega_{0}^{f}} F\left(W_{0}\right) \cdot \frac{\partial \hat{q}}{\partial x_{0}} d x_{0}+\int_{\partial \Omega_{0}^{f}}\left(F\left(W_{0}\right) \cdot n_{0}\right) \cdot \hat{q} d a_{0}=0, \forall \hat{q},
$$

satisfaites par $W_{0}$ au point initial $x_{0}$, on retrouve les équations d'Euler standard:

$$
\begin{aligned}
& \pm \int_{\partial \Omega_{0}^{f}} F\left(W_{0}+\delta W\right) \cdot \frac{\partial \hat{q}}{\partial x_{0}} d x_{0} \\
& +\int_{\partial \Omega_{0}^{f}} \hat{q}\left(x_{0}\right) F\left(W_{0}+\nabla W_{0} \cdot \delta x+\delta W\right) \cdot n d a=0, \forall \hat{q},
\end{aligned}
$$

écrites sur la configuration initiale fixe $\Omega_{0}$, mais avec des termes frontières totalement non standard. De plus, par construction de l'inconnue $\delta W$, l'écriture de la condition d'interface (5) va prendre ici la forme dite de transpiration :

$$
\operatorname{Tr}\left(U^{s}\right)_{\mid \Gamma} \cdot n d a=\operatorname{Tr}\left(U_{0}^{f}\right)_{\mid \Gamma_{0}} . n d a+\operatorname{Tr}\left(\nabla U_{0}^{f} . \delta x+\delta U\right)_{\mid \Gamma_{0}} . n_{0} d a_{0} .
$$

Ces équations (7)-(8) correspondent à quelques simplifications près aux formulations en transpiration utilisées en milieu industriel. Par construction, elles ne sont valides que si le déplacement $\delta x$ est petit, et que si la correction $\delta W$ est aussi petite là où le déplacement est non nul [12].

\section{CONCLUSIONS}

L'article présenté s'intéresse avant tout aux formulations mathématiques et numériques des problèmes d'interaction de fluide-structure en présence de déformations grandes ou modérées. Nous avons présenté quelques formulations de 
base largement utilisées en pratique, ainsi que les problèmes numériques potentiellement associés. Les points difficiles abordés concernent d'abord la consistance du traitement en temps des forces d'interface afin de bien vérifier les principes de conservation d'énergie. Les schémas découplés simplifient la résolution numérique, mais sont potentiellement dangereux à ce niveau. Les schémas couplés permettant une vérification plus automatique de ces principes quand les variations de configuration de la structure sont négligés. Par contre, et c'est le deuxième point abordé, les schémas couplés permettent aussi des fuites ou des gains d'énergie en domaine mobile, et ceci surtout pour des schémas peu précis utilisés sur des grilles peu régulières en temps. Le dernier aspect est une introduction mathématique des équations dites de transpiration qui permettent d'ignorer les déformations de la structure via un changement adéquat des conditions aux limites. Ces notions permettent déjà de bien cibler les stratégies numériques utilisables pour les calculs de fluide structure en temps long, mais leur étude théorique et numérique est encore un peu incomplète dans le cas de problèmes instationnaires rapides.

\section{RÉFÉRENCES}

[1] Kunl. D., Ramm E. (1998), - Generalized Energy Momentum Method for Nonlinear Adaptive Shell Dynamics, submitted to Computer Methods in Applied Mechanics and Engineering.

[2] Dautray R., Lions J.-L. (1984). - Analyse Mathématique et Calcul Numérique pour les Sciences et les Techniques, Collection du Commissariat à l'Energie Atomique, Masson, Paris.

[3] Piperno S., Farhat C., Larrouturou B. (1995). - Partitioned procedures for the transient solution of coupled aeroelastic problems, Computer Methods in Applied Mechanics and Engineering, 124, 79-111.
[4] Le TAllec P. (1994). - Numerical Methods for Nonlinear Three-dimensional Elasticity. Handbook of Numerical Analysis, Vol. III, 465-624.

[5] Le Tallec P., Mouro J. (1999). - Fluid Structure Interaction with Large Structural Displacements, to appear in Computer Methods in Applied Mechanics and Engineering.

[6] Farhat C., Lesoinne M. and Le Tallec P. (1998), - Load and Motion Transfer Algorithms for Fluid/Structure Interaction Problems with Non-Matching Discrete Interfaces : Momentum and Energy Conservation, Optimal Discretization and Application to Aeroelasticity. Comp. Meth. Appl. Mech. Eng., Vol. 157, 1-2, 95-114.

[7] N'Konga B., Guillard H. (1994), - Godunov type method on non-structured meshes for three dimensional moving boundary problems. Comput. Methods Appl. Mech. Eng., 113, pp. 183-204.

[8] Guillard H., Farhat C. (1998). - On the significance of the geometric conservation law for flow computations on moving meshes, Research Report CU-CAS-98-08, Center for Aerospace Structures, University of Colorado, May 1998.

[9] LE TALleE P., MANI S. (1999), - Numerical Analysis of a Linearised Fluid Structure Interaction Problem, Research Report CEREMADE 9917, Université de Paris Dauphine, Mai 1999, à paraitre dans Numerische Mathematik.

[10] Le Tallec P., Mani S. (1999). - Conservation Laws for Fluid Structure Interactions. Proceedings of Fluid Structure Interaction 99, Tron Kvamsdal ed., Trondheim, February 1999.

[11] Le Tallec P., Mouro J. (1998). - Fluid Structure Interaction with Large Structural Displacements, Proceedings of ECCOMAS 98, pp. 1032-1040, K.D. Papailiou, D. Tsahalis, J. Périaux, C. Hirsch, M. Pandolfi, eds, J. Wiley and Sons Publisher.

[12] Fanion T., Fernandez M. A., Le Tallec P. (2000). Deriving Adequate Formulations for Fluid Structure Interaction Problems : from ALE to Transpiration. A paraître dans Revue Européenne Eléments Finis. 